\title{
DETERMINATION OF THORACIC COMPUTERIZED TOMOGRAPHY FINDINGS IN COVID-19 CASES BY DEEP LEARNING
}

\author{
Sua Sumer ${ }^{1}$, Onur Ural ${ }^{1}$, Asli Ural ${ }^{2}$, NAZLIM AKTUĞ DEMIR ${ }^{3}$, Hakan Cebeci ${ }^{1}$, Abidin \\ Kilincer $^{3}$, Fikret Kanat ${ }^{3}$, Ediz Uslu ${ }^{2}$, Mustafa Koplay ${ }^{3}$, Lutfi Saltuk Demir ${ }^{4}$, Ugur Arslan ${ }^{1}$, \\ and Nusret Seher ${ }^{3}$
}

${ }^{1}$ Selcuk University Faculty of Medicine

${ }^{2}$ JotForm Yazilim A.S.

${ }^{3}$ Selcuk University, Faculty of Medicine

${ }^{4}$ Necmettin Erbakan University Faculty of Medicine

August 3, 2021

\begin{abstract}
Aims: The effects of the COVID-19 pandemic continue around the world. Imaging methods play an important role in the diagnosis of COVID-19. The aim of this study was to develop a system that would allow for the distinguishing of lesions at different stages of the disease based on similar signs of other viral diseases and monitoring the emergence, progression, and/or remission of lesions in different areas of the lungs. Methods: For the deep learning (DL) system, the thoracic CT images from 1,382 images were reviewed. These belonged to patients whose SARS-CoV-2 RT-PCR tests turned out positive, were diagnosed with COVID-19, and had signs of lung involvement. Of 1,382 images in the dataset, 180 were assigned for testing and 1,202 were assigned for training. Apart from our dataset, 131 images for internal testing and 1,365 images for external testing were used. The trainings were continued to cover 316,000 steps. Results: Internal and external analyses were used to assess the developed model. The internal analysis success rate was $93.12 \%$. For first external analysis we used 85 images. In the first external analysis we assessed a single CT image of each patient who was in the mixed image lists, and the success rate was found to be $70.31 \%$. In the second external analysis, 645 thoracic CT images of patients diagnosed with COVID-19 and 635 images of another patients who had signs of non-COVID-19 diseases were used. We assessed the thoracic CT images with both COVID-19 and non-COVID-19 disease signs. The success rate in the identification of COVID-19 patients was $88.4 \%$. Conclusion: Special modeling systems developed using DL may help accelerate workflow and making the process easier. This is especially important in cases in which fast and accurate assessment is essential for of a large number of patients, as happens in a pandemic.
\end{abstract}

\section{Hosted file}

COVID-19 DL (Clinical Practice - Main Document).docx available at https://authorea.com/users/ 425898/articles/532742-determination-of-thoracic-computerized-tomography-findings-incovid-19-cases-by-deep-learning 

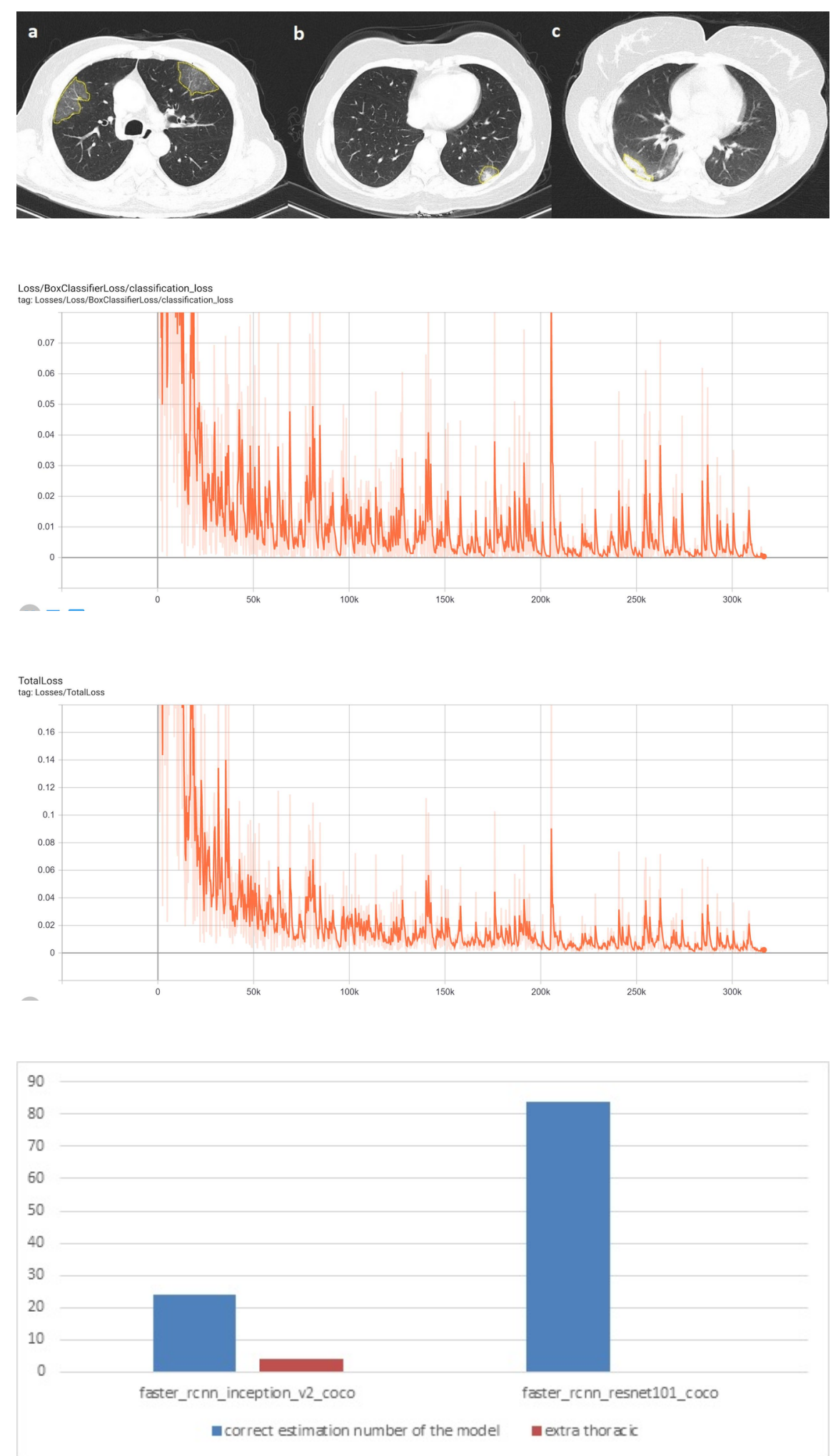

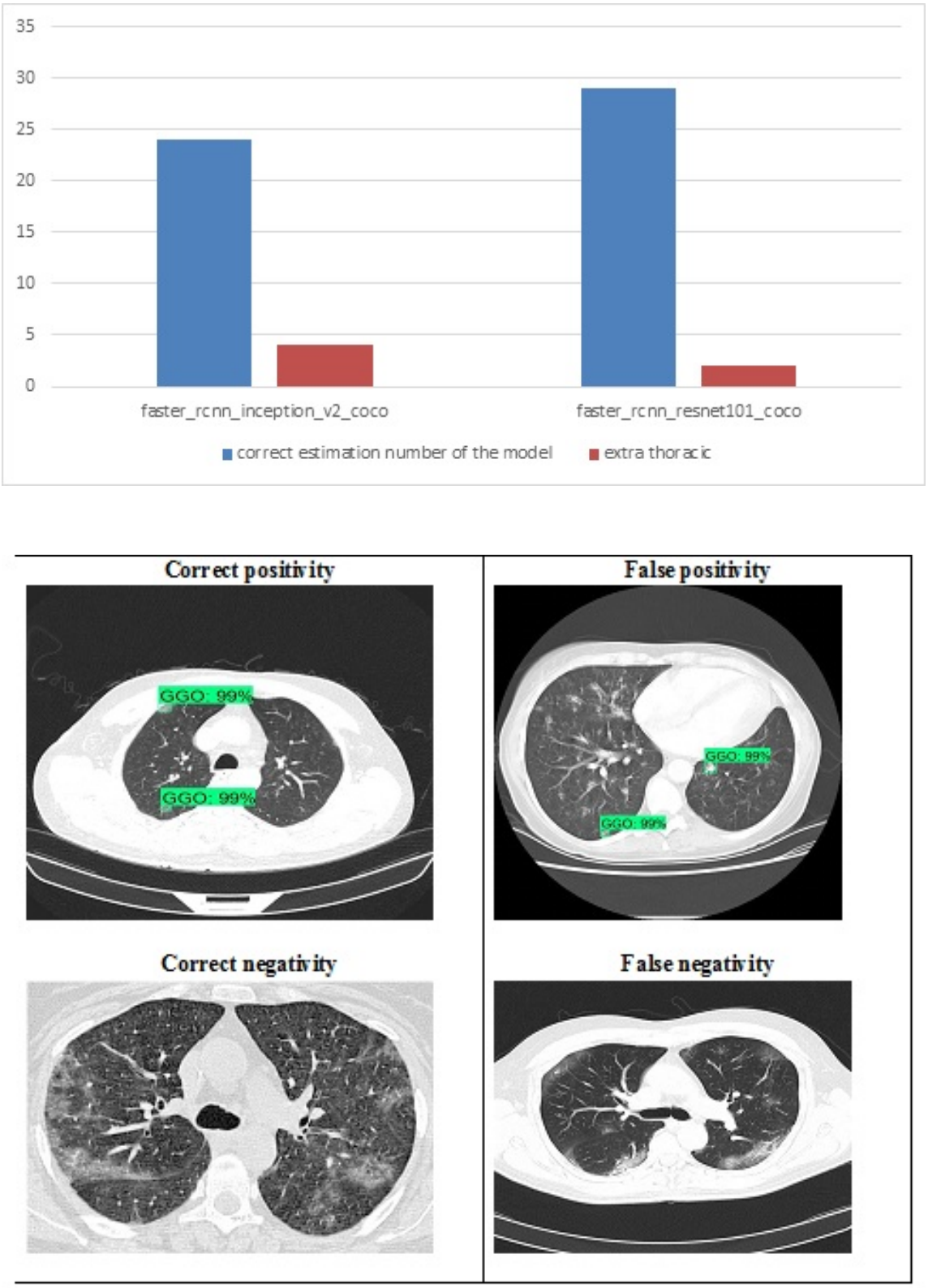

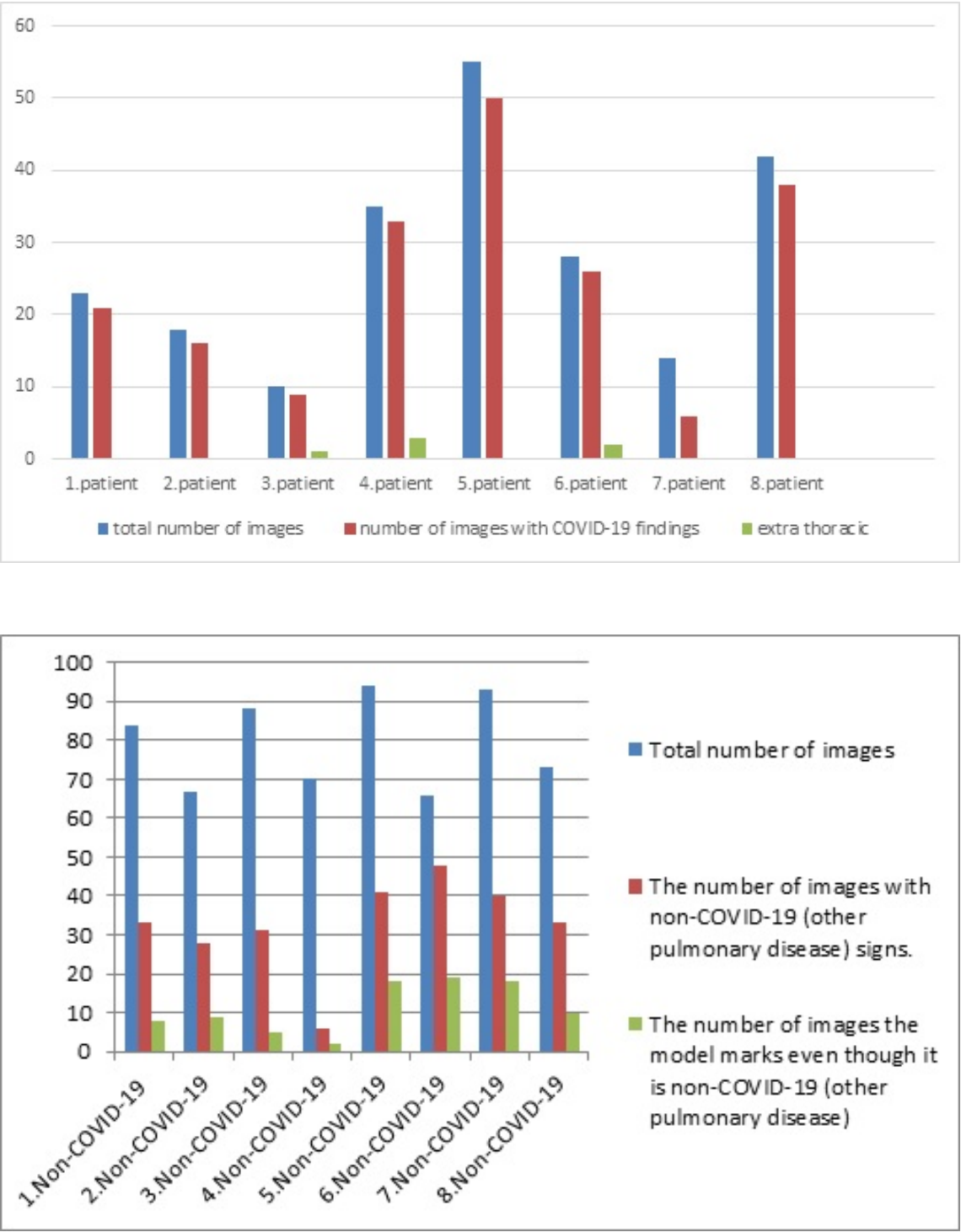